\title{
Delayed phenomenon of loss of stability of solutions in a second-order quasi-linear singularly perturbed boundary value problem with a turning point
}

Zheyan Zhou ${ }^{*}$ and Jianhe Shen

* Correspondence: zzy@fjnu.edu.cn School of Mathematics and Computer Science, Fujian Normal University, Fuzhou 350007, People's Republic of China

\begin{abstract}
Based on the method of differential inequalities, by constructing the upper ad lower solutions suitably, delayed phenomenon of loss of stability of solutions in a secondorder quasi-linear singularly perturbed Dirichlet boundary value problem with a turning point is found in this paper. An illustrating example is performed to verify the obtained results.
\end{abstract}

Keywords: Upper and lower solutions, singular perturbation, turning point, delay of loss of stability

\section{\$1 Introduction}

In real-world applications, there are numerous examples, from biology, chemistry, neurophysiology, fluid dynamics, automation, semiconductor laser, etc., are described in dynamical systems with singular perturbation. The process evolving more than one scale in time and/or space is a typical feature of such type of dynamical systems.

The studies of singular perturbation can be traced back to nineteenth century stimulated greatly by celestial mechanics at that time. The Lindstedt-Poincaré method could be regarded as the first invention to deal with the secular term problems, which is one of the two broad categories of singularly perturbed problems $[1,2]$. Another broad category of singularly perturbed problems is the boundary layer problems $[1,2]$. The idea of boundary layer was proposed by Prandtl in the setting of fluid dynamics and aerodynamics. Matching principle was an invention of Prandtl to obtain uniformly valid asymptotic solutions of boundary layer problems.

In the process of developing the theory of singular perturbation, Tikhonov's limit theory $[3,4]$ and Fenichel's geometric theory $[5,6]$ are two seminal works. Both the two theories tell us that the solutions of singularly perturbed problems tend to the stable solutions of the corresponding reduced problems with the small parameter approaching to zero under the normally hyperbolic condition. Since then, under this essential condition of normal hyperbolicity, the theory of singular perturbation finds applications in many problems including boundary value problems [7], existence of solitons [8], and biological models [9], etc. 
However, there are many practical situations in which the normal hyperbolicity of the reduced solutions lose. That is, in geometrical speaking, there exist turning points on the critical curve. The existence of turning points leads to several new phenomena in singularly perturbed systems such as exchange of stability and delay of exchange of stability [10]. In general speaking, both exchange of stability and delay of exchange of stability have tight relationship with relaxation oscillations and the latter may lead to canards.

Delay of loss (or exchange) of stability is a typical characteristic of canards detected first in singularly perturbed systems before 30 years ago by the technique of nonstandard analysis [11]. Eckhaus [12] applied standard asymptotic analysis and found the canard phenomenon too. From then on, canard has been studied extensively and several methods including matching asymptotic expansion and blow-up, etc., have been developed. Nowadays, it has been well known that canards are not the exotic objects, but occur frequently in a great deal of real-world applications including chemical reactions [13] and neuron dynamics [14] and so on.

An easy and interesting example for explaining canard solutions was provided by O'Malley in $[15,16]$,

$$
\varepsilon y^{\prime}=x y, \quad y(-1)=y_{0},
$$

which is a first-order linear singularly perturbed initial value problem, in which $x=0$ is the turning point. Shchepakina et al. [17] gave also several systems for illustrating canards. However, as far as the authors know, there are rare contributions concerning canards in nonlinear singularly perturbed boundary value problems. In fact, the solution of a second-order linear two-point boundary value problem as follows, contained in the monograph of Kevorkian and Cole [1], is a canard,

$$
\begin{aligned}
& \varepsilon \gamma^{\prime \prime}-x y^{\prime}+y=0,-1 \leq x \leq 1 ; 0<\varepsilon \ll 1, \\
& y(-1)=1, \quad \gamma(1)=2,
\end{aligned}
$$

in which, $x=0$ is the turning point. This canard was approximated by matching asymptotic expansion with the aid of the variational approach.

In this paper, based on the method of differential inequalities, by constructing the upper and lower solutions suitably, delayed phenomenon of loss of stability of solutions in the following second-order quasi-linear Dirichlet boundary value problem with a turning point is studied in details,

$$
\begin{aligned}
& \varepsilon y^{\prime \prime}+y y^{\prime}+y=0, \quad a \leq x \leq b ; \quad 0<\varepsilon \ll 1, \\
& y(a)=A, \\
& y(b)=B,
\end{aligned}
$$

in which, the prime denotes the derivative with respect to $x, 0<\varepsilon \ll 1$ is a small parameter, $a, b, A$, and $B$ are constants with $a<0<b$ and

$$
a+b=0, \quad A+B=0 .
$$

In other words, existence of canard solutions in boundary value problem (1-3) is obtained in this paper. 
The paper is arranged as follows. In the next section, the asymptotic solution of (1-3) is constructed formally. The uniform validity and the error of the asymptotic solution are given in Section 3, which form the main results of the present paper. By the dynamical behavior of the asymptotic solution, we know that the solution of (1-3) approximated by this asymptotic solution has the feature of delay of loss of stability, i.e., it is a canard. In Section 4, an illustrating example is provided for verifying the correctness of the main results in the paper.

Remark 1. If the solution of boundary value problem (1-3) changes sign in the interval $(a, b)$, then it is said that boundary value problem (1-3) has a turning point.

Remark 2. Although there have been many works concentrating on singularly perturbed problems with turning points, however, as far as the authors know, it seems so far that rare works are concerning with canard solutions in quasi-linear singularly perturbed boundary value problems.

\section{\$2 Construction of the asymptotic solution}

Set $\varepsilon=0$ in Equation (1), we obtain the reduced equation

$$
y y^{\prime}+y=0,
$$

which has a family of solutions

$$
u(x)=-x+C,
$$

where $C$ is a constant of integration to be determined and a particular solution $u_{p}(x)$ $\equiv 0$.

Obviously, the trivial solution $u_{p}(x) \equiv 0$ is lack of attraction. Hence, in general, it is not reasonable to expect that there exist the solutions of (1-3) to be attracted by this particular solution.

On the other hand, by direct linear stability analysis, it can be seen that the solutions defined in (6) are attracted for $x<C$ and repelled for $x>C$. Hence, $x=C$ is viewed as a turning point, where $a<C<b$ is assumed. However, in the next section, utilizing the method of upper and lower solutions, we will prove that there exists at least one solution of (1-3) tending to one of the family of solutions (6) on the whole interval $(a, b)$ with $\varepsilon \rightarrow 0$. This is the delayed phenomenon of loss of stability of solutions occurring particularly in singularly perturbed systems with turning points.

The solutions defined in (6) can be regarded as the outer solutions. Generally, they cannot satisfy the boundary conditions (2) and (3). Consequently, there will be two boundary layers at the ended points of the interval. Hence, for obtaining the uniformly valid asymptotic solution, corrections must be performed at the regions of boundary layers.

Introduce a fast time scale,

$$
\tau_{1}=\frac{x-a}{\varepsilon} \in[0,+\infty)
$$

by which, Equation (1) and boundary condition (2) can, respectively, be transformed into the following forms,

$$
\frac{\mathrm{d}^{2} y}{\mathrm{~d} \tau_{1}^{2}}+\gamma \frac{\mathrm{d} y}{\mathrm{~d} \tau_{1}}+\varepsilon y=0
$$


and

$$
\gamma(0)=A \text {. }
$$

Making $\varepsilon \rightarrow 0$ in (7) yields

$$
\frac{\mathrm{d}^{2} y}{\mathrm{~d} \tau_{1}^{2}}+y \frac{\mathrm{d} y}{\mathrm{~d} \tau_{1}}=0
$$

which is solvable. The solution of Equation (9) satisfying condition (8), denoted by $V_{L}\left(\tau_{1}\right)$, can be regarded as the zero-order approximation to the solution of (7) and (8). In other words, $V_{L}\left(\tau_{1}\right)$ is a zero-order approximation to the left boundary layer. Of course, at present, this zero-order approximation contains a constant to be determined by matching.

$$
\begin{gathered}
\text { Let } \frac{d V_{L}}{\mathrm{~d} \tau_{1}}=P \text {, then } \frac{\mathrm{d}^{2} V_{L}}{\mathrm{~d} \tau_{1}^{2}}=\frac{\mathrm{d} P}{\mathrm{~d} \tau_{1}}=P \frac{\mathrm{d} P}{\mathrm{~d} V_{L}} \text {. Accordingly, Equation (9) is reduced to } \\
P \frac{\mathrm{d} P}{\mathrm{~d} V_{L}}+P V_{L}=0
\end{gathered}
$$

admitting $P \equiv 0$ which is discarded, and

$$
\frac{\mathrm{d} P}{\mathrm{~d} V_{L}}=-V_{L},
$$

which finally yields

$$
P=-\frac{V_{L}^{2}}{2}+C_{1}
$$

i.e.,

$$
\frac{\mathrm{d} V_{L}}{\mathrm{~d} \tau_{1}}=-\frac{V_{L}^{2}}{2}+C_{1},
$$

where $C_{1}$ is a nonzero constant of integration.

Denote $2 C_{1}=a_{1}^{2}$, in which $a_{1} \in R$. Hence, $C_{1}>0$ is meant. Consequently, Equation (12) can be rewritten as

$$
\frac{\mathrm{d} V_{L}}{V_{L}^{2}-a_{1}^{2}}=-\frac{1}{2} \mathrm{~d} \tau_{1} .
$$

Integrating both sides of Equation (13) yields

$$
\frac{V_{L}-a_{1}}{V_{L}+a_{1}}= \pm M_{1} e^{-a_{1} \tau_{1}}
$$

where $M_{1}>0$ is a constant of integration. There are two cases to be discussed.

Case I: $\left|V_{L}\right|>\left|a_{1}\right|$. In this case, we have

$$
\begin{aligned}
V_{L}\left(\tau_{1}\right) & =a_{1} \frac{1+M_{1} e^{-a_{1} \tau_{1}}}{1-M_{1} e^{-a_{1} \tau_{1}}}=a_{1} \frac{1+e^{-a_{1}\left(\tau_{1}+d_{1}\right)}}{1-e^{-a_{1}\left(\tau_{1}+d_{1}\right)}}=a_{1} \frac{e^{\frac{a_{1}}{1}\left(\tau_{1}+d_{1}\right)}+e^{-\frac{a_{1}}{2}\left(\tau_{1}+d_{1}\right)}}{e^{\frac{a_{1}}{1}\left(\tau_{1}+d_{1}\right)}-e^{-\frac{a_{1}}{2}\left(\tau_{1}+d_{1}\right)}} \\
& =a_{1} \operatorname{coth}\left[\frac{a_{1}}{2}\left(\tau_{1}+d_{1}\right)\right],
\end{aligned}
$$


which is a hyperbolic coth function with

$$
V_{L}^{\prime}\left(\tau_{1}\right)=-\frac{2 a_{1}^{2}}{\varepsilon} \frac{M_{1} e^{-a_{1} \tau_{1}}}{\left(1-M_{1} e^{-a_{1} \tau_{1}}\right)^{2}}=-\frac{a_{1}^{2}}{2 \varepsilon} \operatorname{csch}^{2}\left[\frac{a_{1}}{2}\left(\tau_{1}+d_{1}\right)\right]<0,
$$

in which, $d_{1}$ is a constant determined by

$$
M_{1}=e^{-a_{1} d_{1}} .
$$

Case II: $\left|V_{L}\right|<\left|a_{1}\right|$. In this case,

$$
V_{L}\left(\tau_{1}\right)=a_{1} \frac{1-M_{1} e^{-a_{1} \tau_{1}}}{1+M_{1} e^{-a_{1} \tau_{1}}}=a_{1} \tanh \left[\frac{a_{1}}{2}\left(\tau_{1}+d_{1}\right)\right] .
$$

Direct calculations show that

$$
V_{L^{\prime}}\left(\tau_{1}\right)=\frac{2 a_{1}^{2}}{\varepsilon} \frac{M_{1} e^{-a_{1} \tau_{1}}}{\left(1+M_{1} e^{-a_{1} \tau_{1}}\right)^{2}}=\frac{a_{1}^{2}}{2 \varepsilon} \operatorname{sech}^{2}\left[\frac{a_{1}}{2}\left(\tau_{1}+d_{1}\right)\right]>0,
$$

where $d_{1}$ is defined in Equation (15).

Obviously, it follows from Equations (14) and (16) that the function $V_{L}\left(\tau_{1}\right)$ given in cases I and II is, respectively, the monotone decreasing and increasing functions.

Matching between the outer solutions and the left boundary layer correction requires that

$$
V_{L}(+\infty)=u(a) .
$$

Thus, if $a_{1}<0$, since

$$
\lim _{\tau_{1} \rightarrow+\infty} a_{1} \operatorname{coth}\left[\frac{a_{1}}{2}\left(\tau_{1}+d_{1}\right)\right]=\lim _{\tau_{1} \rightarrow+\infty} a_{1} \tanh \left[\frac{a_{1}}{2}\left(\tau_{1}+d_{1}\right)\right]=-a_{1},
$$

then

$$
-a_{1}=u(a)=-a+C>0 .
$$

If $a_{1}>0$, since

$$
\lim _{\tau_{1} \rightarrow+\infty} a_{1} \operatorname{coth}\left[\frac{a_{1}}{2}\left(\tau_{1}+d_{1}\right)\right]=\lim _{\tau_{1} \rightarrow+\infty} a_{1} \tanh \left[\frac{a_{1}}{2}\left(\tau_{1}+d_{1}\right)\right]=a_{1},
$$

then

$$
a_{1}=u(a)=-a+C>0 .
$$

Now, it can be seen that both the $a_{1}<0$ and $a_{1}>0$ cases are possible for matching. Therefore, without loss of generality, the $a_{1}>0$ case can be adopted. Consequently, we have the hyperbolic coth function

$$
V_{L}\left(\tau_{1}\right)=u(a) \frac{1+M_{1} e^{-u(a) \tau_{1}}}{1-M_{1} e^{-u(a) \tau_{1}}}=u(a) \operatorname{coth}\left[\frac{u(a)}{2}\left(\tau_{1}+d_{1}\right)\right]
$$

and the hyperbolic tanh function

$$
V_{L}\left(\tau_{1}\right)=u(a) \frac{1-M_{1} e^{-u(a) \tau_{1}}}{1+M_{1} e^{-u(a) \tau_{1}}}=u(a) \tanh \left[\frac{u(a)}{2}\left(\tau_{1}+d_{1}\right)\right] .
$$

Both of them are possible to be the left boundary layer correction. 
By setting $\tau_{1}=0$ in Equations (17) and (18) and taking Equation (8) into account, we obtain from (17) and (18), respectively, that

$$
A=u(a) \frac{1+M_{1}}{1-M_{1}}
$$

and

$$
A=u(a) \frac{1-M_{1}}{1+M_{1}},
$$

by which, the constant $M_{1}$ is determined, i.e., equivalently, the constant $d_{1}$ in (15) is determined. Till now, $V_{L}\left(\tau_{1}\right)$ defined in (17) and (18) have been determined completely.

Similarly, matching between the outer solutions and the right boundary layer correction requires that

$$
V_{R}(-\infty)=u(b) .
$$

In the same way, two boundary layer functions possible to be the corrections on the right turn out to be

$$
V_{R}\left(\tau_{2}\right)=u(b) \frac{1+M_{2} e^{-u(b) \tau_{2}}}{1-M_{2} e^{-u(b) \tau_{2}}}=u(b) \operatorname{coth}\left[\frac{u(b)}{2}\left(\tau_{2}+d_{2}\right)\right]
$$

and

$$
V_{R}\left(\tau_{2}\right)=u(b) \frac{1-M_{2} e^{-u(b) \tau_{2}}}{1+M_{2} e^{-u(b) \tau_{2}}}=u(b) \tanh \left[\frac{u(b)}{2}\left(\tau_{2}+d_{2}\right)\right],
$$

in which, $u(b)=-b+C<0$,

$$
\tau_{2}=\frac{x-b}{\varepsilon} \in(-\infty, 0]
$$

is another fast time scale, and $d_{2}$ is a constant to be determined by the following equality

$$
M_{2}=e^{-u(b) d_{2}} .
$$

Similarly, the function $V_{R}\left(\tau_{2}\right)$ defined in Equations (21) and (22) is, respectively, the monotone decreasing and increasing functions.

Finally, like the deductions of (19) and (20), we have, respectively, that

$$
B=u(b) \frac{1+M_{2}}{1-M_{2}}
$$

and

$$
B=u(b) \frac{1-M_{2}}{1+M_{2}}
$$

by which, the constant $M_{2}$, i.e., the constant $d_{2}$ is determined. Consequently, the function $V_{R}\left(\tau_{2}\right)$ in Equations (21) and (22) is completely known.

Now, it has been clearly known that, near the left- and right-ended points of the interval, there both have two candidates, possible to be the boundary layer corrections. Which pair of the hyperbolic functions in Equations (17-18) and (21-22) is chosen 
depending on the practical situations like the boundary conditions. In the following of the paper, we will show that the hyperbolic coth functions defined in Equations (17) and (21) must be selected to be the left and right boundary layer corrections, respectively.

Consequently, so far the formally asymptotic solution is given by

$$
y_{\text {asy }}(x, \varepsilon)=u(x)+V_{L}\left(\tau_{1}\right)+V_{R}\left(\tau_{2}\right),
$$

in which, $u(t), V_{L}\left(\tau_{1}\right)$, and $V_{R}\left(\tau_{2}\right)$ are defined in Equations $(6,17)$, and (21), respectively, and the constant $C$ in Equation (6) will be determined later. In the following section, based on the theory of differential inequalities, by constructing the upper and lower solutions suitably, we will prove that this asymptotic solution is uniformly valid with certain order. Consequently, by the dynamical behavior of the asymptotic solution (25), delay loss of stability of solution in (1-3) can be seen, i.e., existence of canard solutions in (1-3) is known and this canard is approximated uniformly by the asymptotic solution (25).

\section{\$3 A lemma and the main results}

To prove the main results of the current paper, the following lemma is needed.

Lemma 1 [18] Consider second-order nonlinear boundary value problems with Dirichlet boundary conditions,

$$
\begin{cases}y^{\prime \prime}=f\left(x, y, y^{\prime}\right), & x \in(a, b) \\ y(a)=A, & y(b)=B\end{cases}
$$

in which $a, b, A$, and $B$ are constants.

For this boundary value problem, if the following conditions hold,

(1) there exist the upper and lower solutions, i.e., there are functions $\beta(x), \alpha(x) \in C^{2}$ $[a, b]$ with $\beta(x) \geq \alpha(x)$ such that

$$
\begin{aligned}
& \beta^{\prime \prime} \leq f\left(x, \beta, \beta^{\prime}\right), \quad x \in(a, b), \\
& \beta(a) \geq A, \quad \beta(b) \geq B
\end{aligned}
$$

and

$$
\begin{aligned}
& \alpha^{\prime \prime} \geq f\left(x, \alpha, \alpha^{\prime}\right), \quad x \in(a, b), \\
& \alpha(a) \leq A, \quad \alpha(b) \leq B,
\end{aligned}
$$

(2) the function $f\left(x, y, y^{\prime}\right)$ satisfies the Nagumo condition with respect to $\beta(t)$ and $\alpha$ $(t)$, then there exists at least one solution $y(x) \in C^{2}[a, b]$ with the following estimate:

$$
\alpha(x) \leq \gamma(x) \leq \beta(x), \quad x \in[a, b] .
$$

Based on Lemma 1, we turn to prove the following theorems.

Theorem 1 There exists at least one solution of boundary value problem (1-3) such that

$$
\left|\gamma(x, \varepsilon)-\gamma_{\text {asy }}(x, \varepsilon)\right| \leq \gamma \varepsilon, \quad x \in[a, b],
$$

where $\gamma$ is a positive constant, $y_{\text {asy }}(x, \varepsilon)$ is given by Equation $(25)$ in which

$$
u(x)=-x,
$$

i.e., $C=0$ in Equation (6) is determined. 
By Theorem 1 and the dynamical behavior of $y_{\text {asy }}(x, \varepsilon)$, the following Theorem 2 can be concluded directly.

Theorem 2 There exist at least one solution of boundary value problem (1-3) with the following asymptotic behavior:

$$
\lim _{\varepsilon \rightarrow 0} \gamma(x, \varepsilon)=-x, \quad x \in(a, b) .
$$

Theorems 1 and 2 together mean Theorem 3 as follows.

Theorem 3 Boundary value problem (1-3) has at least one canard solution, whose zero-order approximation is given by Equation (25).

Proof of Theorem 1 Define the upper and lower solutions as follows:

$$
\beta(x, \varepsilon)=u(x)+V_{L}\left(\tau_{1}\right)+V_{R}\left(\tau_{2}\right)+x^{4} \gamma \varepsilon
$$

and

$$
\alpha(x, \varepsilon)=u(x)+V_{L}\left(\tau_{1}\right)+V_{R}\left(\tau_{2}\right)-x^{4} \gamma \varepsilon,
$$

in which, $\gamma$ is a positive constant.

Since the right-hand side function in Equation (1) satisfies the Nagumo condition, thus, to obtain Theorem 1, it is left to verify that the upper and lower solutions (31) and (32) satisfy the condition (1) in Lemma 1.

Firstly, we prove the following inequality:

$$
\varepsilon \beta^{\prime \prime}+\beta \beta^{\prime}+\beta \leq 0 .
$$

In fact,

$$
\begin{aligned}
\varepsilon & \beta^{\prime \prime}+\beta \beta^{\prime}+\beta \\
= & \varepsilon\left(\frac{\ddot{V}_{L}}{\varepsilon^{2}}+\frac{\ddot{V}_{R}}{\varepsilon^{2}}+12 x^{2} \gamma \varepsilon\right)+\left(u(x)+V_{L}+V_{R}+x^{4} \gamma \varepsilon\right)\left(u^{\prime}(x)+\frac{\dot{V}_{L}}{\varepsilon}+\frac{\dot{V}_{R}}{\varepsilon}+4 x^{3} \gamma \varepsilon\right) \\
& +u(x)+V_{L}+V_{R}+x^{4} \gamma \varepsilon \\
= & 12 x^{2} \gamma \varepsilon^{2}+\left(u(x)+V_{R}+x^{4} \gamma \varepsilon\right) \frac{\dot{V}_{L}}{\varepsilon}+\left(u(x)+V_{L}+x^{4} \gamma \varepsilon\right) \frac{\dot{V}_{R}}{\varepsilon} \\
& +4 x^{3} \gamma \varepsilon\left(u(x)+V_{L}+V_{R}+x^{4} \gamma \varepsilon\right) \\
= & \frac{1}{\varepsilon}\left[\left(u(x)+V_{R}+x^{4} \gamma \varepsilon\right) \dot{V}_{L}+\left(u(x)+V_{L}+x^{4} \gamma \varepsilon\right) \dot{V}_{R}\right. \\
& \left.\quad+4 x^{3} \gamma \varepsilon^{2}\left(u(x)+V_{L}+V_{R}+x^{4} \gamma \varepsilon\right)+12 x^{2} \gamma \varepsilon^{3}\right],
\end{aligned}
$$

in which as well as in the following of the paper, the prime and the dot always denote the derivations with respect to the slow scale $x$ and the fast scales $\tau_{1}, \tau_{2}$, respectively.

We want to prove that the quantity defined in Equation (30) is not positive. The proof is completed by dividing the interval $[a, b]$ into five parts.

Part I. $x \in\left[a, a+\delta_{1}\right)$, where $\delta_{1}>0$ is a sufficiently small constant independent of $\varepsilon$. In this case, it can be deduced from Equation (17) that

$$
\left.V_{L}\left(\tau_{1}\right)\right|_{x=a}=V_{L}(0)=A \text { and }\left.\dot{V}_{L}\left(\tau_{1}\right)\right|_{x=a}=\dot{V}_{L}(0)=-\frac{2 u^{2}(a) M_{1}}{\left(1-M_{1}\right)^{2}}
$$


which are both constants. Similarly, we can derive from Equation (21) that

$$
V_{R}(a)=u(b) \frac{1+M_{2} e^{-u(b) \frac{a-b}{\varepsilon}}}{1-M_{2} e^{-u(b) \frac{a-b}{\varepsilon}}}=u(b)+u(b) \frac{2 M_{2} e^{-u(b) \frac{a-b}{\varepsilon}}}{1-M_{2} e^{-u(b) \frac{a-b}{\varepsilon}}}=u(b)+\mathrm{O}\left(e^{-u(b) \frac{a-b}{\varepsilon}}\right),
$$

in which, for $\varepsilon$ sufficiently small, $\mathrm{O}\left(e^{-u(b) \frac{a-b}{\varepsilon}}\right)$ denotes a quantity that is exponentially small and negative, and

$$
\dot{V}_{R}(a)=-\frac{2 u^{2}(b) M_{2} e^{-u(b) \frac{a-b}{\varepsilon}}}{\left(1-M_{2} e^{-u(b) \frac{a-b}{\varepsilon}}\right)^{2}}
$$

which is a exponential small quantity too.

Substituting Equations (31-33) into Equation (30) and taking $a+b=0$ into account yields

$$
\begin{aligned}
& {\left[\left(u(x)+V_{R}+x^{4} \gamma \varepsilon\right) \dot{V}_{L}+\left(u(x)+V_{L}+x^{4} \gamma \varepsilon\right) \dot{V}_{R}\right.} \\
& \left.\quad+4 x^{3} \gamma \varepsilon^{2}\left(u(x)+V_{L}+V_{R}+x^{4} \gamma \varepsilon\right)+12 x^{2} \gamma \varepsilon^{3}\right]\left.\right|_{x=a} \\
& =-\left[2 C+\mathrm{O}\left(e^{-u(b) \frac{a-b}{\varepsilon}}\right)+a^{4} \gamma \varepsilon\right] \frac{2 u^{2}(a) M_{1}}{\left(1+M_{1}\right)^{2}}-\left(-a+C+A+a^{4} \gamma \varepsilon\right) \frac{2 u^{2}(b) M_{2} e^{-u(b) \frac{a-b}{\varepsilon}}}{\left(1-M_{2} e^{-u(b) \frac{a-b}{\varepsilon}}\right)^{2}} \\
& \quad+4 a^{3} \gamma \varepsilon^{2}\left[2 C+A+\mathrm{O}\left(e^{-u(b) \frac{a-b}{\varepsilon}}\right)+a^{4} \gamma \varepsilon\right]+12 a^{2} \gamma \varepsilon^{3} .
\end{aligned}
$$

By comparing the order of the four parts in Equation (34), we can find that, for $\varepsilon$ sufficiently small, the sign of Equation (34) is determined by its first part, i.e.,

$$
-\left[2 C+\mathrm{O}\left(e^{-u(b) \frac{a-b}{\varepsilon}}\right)+a^{4} \gamma \varepsilon\right] \frac{2 u^{2}(a) M_{1}}{\left(1-M_{1}\right)^{2}} .
$$

Hence, if the constant $C$ in Equation (35) is chosen such that

$$
C \geq 0 \text {, }
$$

then

$$
-\left[2 C+\mathrm{O}\left(e^{-u(b) \frac{a-b}{\varepsilon}}\right)+a^{4} \gamma \varepsilon\right] \frac{2 u^{2}(a) M_{1}}{\left(1-M_{1}\right)^{2}}<0 .
$$

Consequently, when $x=a$, the quantity defined in (30) is negative if the inequality (36) holds and $\varepsilon$ is sufficiently small. Hence, there exists a sufficiently small constant $\delta_{1}$ $>0$ independent of $\varepsilon$ such that the quantity defined in (30) is negative for $x \in[a, a+$ $\left.\delta_{1}\right)$.

On the contrary, we can see that when the following differential inequality to be proved,

$$
\varepsilon \alpha^{\prime \prime}+\alpha \alpha^{\prime}+\alpha \geq 0,
$$

in which, $\alpha(x, \varepsilon)$ is defined in Equation (28), it is required that

$$
C \leq 0 \text {. }
$$


Accordingly, the inequalities (36) and (38) together yield

$$
C=0 .
$$

Therefore, in what follows, $C=0$ is set in Equation (6). Thus, $u(x)=-x$ turns out to be the reduced solution.

Part II. $x=0$.

In this case, since the boundary values in (2-3) satisfy

$$
A+B=0,
$$

it then follows from Equations (19) and (23) that

$$
0=-a \frac{1+M_{1}}{1-M_{1}}-b \frac{1+M_{2}}{1-M_{2}}=b\left(\frac{1+M_{1}}{1-M_{1}}-\frac{1+M_{2}}{1-M_{2}}\right)=2 b \frac{M_{1}-M_{2}}{\left(1-M_{1}\right)\left(1-M_{2}\right)},
$$

in which, $a=-b$ has been noted, which finally implies that

$$
M_{1}=M_{2} \text {. }
$$

Consequently, by setting $x=0$ in Equation (30), one gets

$$
\begin{aligned}
& \frac{1}{\varepsilon}\left[\left(u(0)+V_{R}\left(\frac{-b}{\varepsilon}\right)\right) \dot{V}_{L}\left(\frac{-a}{\varepsilon}\right)+\left(u(0)+V_{L}\left(\frac{-a}{\varepsilon}\right)\right) \dot{V}_{R}\left(\frac{-b}{\varepsilon}\right)\right] \\
& =\frac{1}{\varepsilon}\left[V_{R}\left(\frac{-b}{\varepsilon}\right) \dot{V}_{L}\left(\frac{-a}{\varepsilon}\right)+V_{L}\left(\frac{-a}{\varepsilon}\right) \dot{V}_{R}\left(\frac{-b}{\varepsilon}\right)\right] \\
& =\frac{1}{\varepsilon}\left[-2 a^{2} b \frac{1+M_{2} e^{\frac{-b^{2}}{\varepsilon}}}{1-M_{2} e^{\frac{-b^{2}}{\varepsilon}}} \frac{M_{1} e^{\frac{-a^{2}}{\varepsilon}}}{\left(1+M_{1} e^{\frac{-a^{2}}{\varepsilon}}\right)^{2}}-2 a b^{2} \frac{1+M_{1} e^{\frac{-a^{2}}{\varepsilon}}}{1-M_{1} e^{\frac{-a^{2}}{\varepsilon}}} \frac{M_{2} e^{\frac{-b^{2}}{\varepsilon}}}{\left(1+M_{2} e^{\frac{-b^{2}}{\varepsilon}}\right)^{2}}\right] \\
& =0,
\end{aligned}
$$

in which, $a=-b$ and $M_{1}=M_{2}$ have been taken into account. Thus, when $x=0$, the inequality (29) holds.

Part III. $x \in\left[a=\delta_{1}, 0\right]$.

Taking the cases in Parts I and II into account, if the inequality (29) does not hold uniformly in this region, then there must be at least one point $x^{*} \in\left(a=\delta_{1}, 0\right)$ such that

$$
H\left(x^{*}, \varepsilon\right)>0, H^{\prime}\left(x^{*}, \varepsilon\right)=0 \text { and } H^{\prime \prime}\left(x^{*}, \varepsilon\right) \leq 0,
$$

in which

$$
\begin{aligned}
H(x, \varepsilon)= & \frac{1}{\varepsilon}\left[\left(-x+V_{R}+x^{4} \gamma \varepsilon\right) \dot{V}_{L}+\left(-x+V_{L}+x^{4} \gamma \varepsilon\right) \dot{V}_{R}\right. \\
& \left.+4 x^{3} \gamma \varepsilon^{2}\left(-x+V_{L}+V_{R}+x^{4} \gamma \varepsilon\right)+12 x^{2} \gamma \varepsilon^{3}\right] .
\end{aligned}
$$

However, for $\varepsilon$ sufficiently small, since $\dot{V}_{L}, \dot{V}_{R}$ and $V_{L}+V_{R}$ are exponentially small in this region, thus, it can be shown by direct calculations that

$$
H^{\prime}(x *, \varepsilon)=\varepsilon\left[-12 \gamma\left(x^{*}\right)^{3}+O(\varepsilon)\right]>0,
$$

which is a contradiction.

Part IV. $x \in\left(b-\delta_{2}, b\right]$, where $\delta_{2}>0$ is a sufficiently small constant independent of $\varepsilon$. 
In this region, the proof of the inequality (29) is parallel to Part I completely. Like the deductions of Equations (31-33), the values of $\left.V_{L}\left(\tau_{1}\right)\right|_{x=b},\left.\dot{V}_{L}\left(\tau_{1}\right)\right|_{x=b},\left.V_{R}\left(\tau_{2}\right)\right|_{x=b}$, and $\left.\dot{V}_{R}\left(\tau_{2}\right)\right|_{x=b}$ can be calculated. Consequently, we can see that, when $x=b$, the other parts in Equation (30) are the higher-order small quantities compared with its second part. Thus, the sign of Equation (30) is determined by its second part, which is a negative quantity. Accordingly, the inequality (29) is proved.

Part V. $x \in\left(0, b-\delta_{2}\right.$, , In this region, the proof of the inequality (29) is parallel to Part III completely.

So far the proof of the differential inequality (29) has been finished for $x \in[a, b]$. In the same way, the differential inequality (37) can be proved.

In what follows, we turn to prove the inequalities on the boundaries. For $\varepsilon$ sufficiently small, we have

$$
\begin{aligned}
\beta(a, \varepsilon) & =u(a)+V_{L}(0)+V_{R}\left(\frac{a-b}{\varepsilon}\right)+a^{4} \gamma \varepsilon \\
& =u(a)+A+u(b)+\mathrm{O}\left(e^{-u(b) \frac{a-b}{\varepsilon}}\right)+a^{4} \gamma \varepsilon \\
& =A+\mathrm{O}\left(e^{\frac{b(a-b)}{\varepsilon}}\right)+a^{4} \gamma \varepsilon \\
& \geq A \geq \alpha(a, \varepsilon)=u(a)+V_{L}(0)+V_{R}\left(\frac{a-b}{\varepsilon}\right)-a^{4} \gamma \varepsilon
\end{aligned}
$$

in which, $u(a)=-a, u(b)=-b$, and $a+b=0$ have been used.

Similarly, it can be proved that

$$
\beta(b, \varepsilon) \geq B \geq \alpha(b, \varepsilon) .
$$

Therefore, according to Lemma 1, we have

$$
\alpha(x, \varepsilon) \leq \gamma(x, \varepsilon) \leq \beta(x, \varepsilon), \quad x \in[a, b],
$$

and accordingly, Theorem 1 is derived.

Remark 3. From the proof of Theorem 1, we know that the construction of the upper and lower solutions defined in (27) and (28), respectively, is essential. The error term $x^{4} \gamma \varepsilon$ introduced in (27) and (28) seems necessary for discussing the existence of canard solutions in singularly perturbed problems (1-3).

\section{§ 4 An illustrating example}

Consider a second-order quasi-linear singularly perturbed Dirichlet boundary value problem as follows,

$$
\begin{aligned}
& \varepsilon y^{\prime \prime}+y y^{\prime}+y=0, \quad-1<x<1 ; 0<\varepsilon \ll 1, \\
& \gamma(-1)=2, \\
& \gamma(1)=-2
\end{aligned}
$$

in which, the boundary points and the boundary values satisfy the conditions (4).

According to Theorem 1, the uniformly valid zero-order asymptotic solution of this boundary value problem is given by 


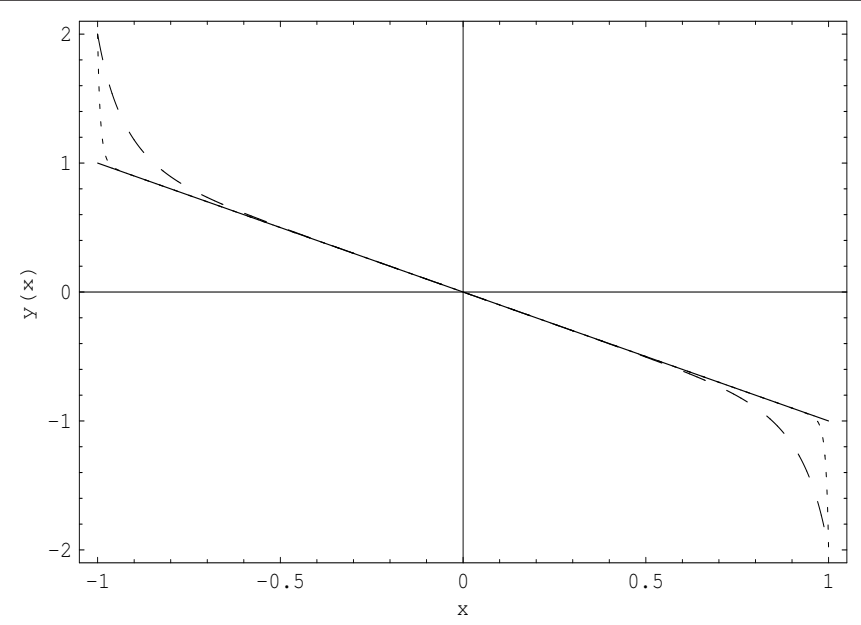

Figure 1 The reduced solution and the asymptotic solutions. Solid line The reduced solution; dashing line the asymptotic solution with $\varepsilon=0.1$; dotted line the asymptotic solution with $\varepsilon=0.01$.

$$
y_{\text {asy }}(x, \varepsilon)=-x+\frac{1+M_{1} e^{\frac{-1-x}{\varepsilon}}}{1-M_{1} e^{\frac{-1-x}{\varepsilon}}}-\frac{1+M_{2} e^{\frac{x-1}{\varepsilon}}}{1-M_{2} e^{\frac{x-1}{\varepsilon}}},
$$

in which, $M_{1}$ and $M_{2}$ are, respectively, determined by

$$
2=\frac{1+M_{1}}{1-M_{1}}
$$

and

$$
-2=-\frac{1+M_{2}}{1-M_{2}}
$$

Consequently,

$$
M_{1}=M_{2}=\frac{1}{3}
$$

are derived.

Substituting Equation (42) into (41) yields

$$
y_{\text {asy }}(x, \varepsilon)=-x+\frac{1+\frac{1}{3} e^{\frac{-1-x}{\varepsilon}}}{1-\frac{1}{3} e^{\frac{-1-x}{\varepsilon}}}-\frac{1+\frac{1}{3} e^{\frac{x-1}{\varepsilon}} \mid}{1-\frac{1}{3} e^{\frac{x-1}{\varepsilon}}} .
$$

The asymptotic solution (43) is simulated in Figure 1 with different values of $\varepsilon$. In the figure, the solid, dashing, and dotted lines represent, respectively, the reduced solution, the asymptotic solutions with $\varepsilon=0.1$ and $\varepsilon=0.01$. From this figure, we can see that

(1) delayed phenomenon of loss of stability of solutions really occurs, that is, existence of canards in this boundary value problem is verified. This canard solution is approximated by (43) with the accuracy of zero-order; 
(2) with $\varepsilon \rightarrow 0$, the asymptotic solution approaches more and more to the reduced solution in the whole interval $(a, b)$. Therefore, the zero-order approximation is sufficiently accurate for the small $\varepsilon$.

\section{Authors' contributions}

The authors wrote this article in collaboration and with same responsibility. All authors read and approved the final manuscript.

\section{Competing interests}

The authors declare that they have no competing interests.

Received: 6 March 2011 Accepted: 14 October 2011 Published: 14 October 2011

\section{References}

1. Kevorkian, JK, Cole, JD: Perturbation Methods in Applied Mathematics. Springer, New York (1981)

2. Verhulst, F: Methods and Applications of Singular Perturbations: Boundary Layers and Multiple Timescales Dynamics. Springer, New York (2005)

3. Tikhonov, AN: On the dependence of solutions of differential equations on a small parameter. Math. Sb. 73, 575-586 (1952)

4. Tikhonov, AN: Systems of differential equations containing a small parameter. Math. Sb. 64, 193-204 (1948)

5. Fenichel, N: Geometric singular perturbation theory for ordinary equations. J. Diff. Equs. 31, 53-98 (1979)

6. Christopher, KRT: Geometric singular perturbation theory. Lecture Notes Math. 1609, 44-118 (1995)

7. Lin, XB: Heteroclinic bifurcation and singularly perturbed boundary value problems. J. Diff. Equs. 84, 319-382 (1990)

8. Beck, M, Doelman, A, Kaper, TJ: A geometric construction of traveling waves in a bioremediation model. J. Nonlinear Sci. 16, 329-349 (2006)

9. Hek, G: Geometric singular perturbation theory in biological practice. J. Math. Biol. 60, 347-386 (2010)

10. Butuzov, VF, Nefedov, NN, Schneider, KR: Singularly perturbed problems in cases of exchange of stabilities. J. Math. Sci. 1210, 1973-2079 (2004)

11. Callot, JL, Diener, F, Diener, M: Le Problème de la "chasse au canard". C. R. Acad. Sci. Paris. 286, 1059-1061 (1978)

12. Eckhaus, W: Relaxation oscillations including a standard chase on French ducks. Lecture Notes Math. 985, 449-494 (1983)

13. Xie, F, Han, M, Zhang, W: Canard phenomena in oscillations of a surface oxidation reaction. J. Nonlinear Sci. 15, 363-386 (2005)

14. Horacio, G., et al: A Canard mechanics for localization in systems of globally coupled oscillators. SIAM J. Appl. Math. 63, 1998-2019 (2003)

15. O'Malley, RE Jr: Singular Perturbation Methods for Ordinary Differential Equations. Springer, New York (1991)

16. Lin, P, O'Malley, RE Jr: The numerical solution of a challenging class of turning problems. SIAM J. Sci. Comput. 25, 927-941 (2003)

17. Shchepakina, E, Sobolev, V: Integral manifolds, canards and black swans. Nonlinear Anal. TMA. 44, $897-908$ (2001)

18. Chang, KW, Howes, FA: Nonlinear Singular Perturbation Phenomena: Theory and Application. Springer, New York (1983)

doi:10.1186/1687-2770-2011-35

Cite this article as: Zhou and Shen: Delayed phenomenon of loss of stability of solutions in a second-order quasi-linear singularly perturbed boundary value problem with a turning point. Boundary Value Problems 2011 2011:35.

\section{Submit your manuscript to a SpringerOpen ${ }^{\circ}$ journal and benefit from:}

Convenient online submission

- Rigorous peer review

- Immediate publication on acceptance

- Open access: articles freely available online

- High visibility within the field

- Retaining the copyright to your article

Submit your next manuscript at $\mathbf{s p r i n g e r o p e n . c o m ~}$ 